\title{
Inspiration Is the Major Regulator of Human CSF Flow
}

\author{
Steffi Dreha-Kulaczewski, ${ }^{1}$ Arun A. Joseph, ${ }^{2,3}$ Klaus-Dietmar Merboldt, ${ }^{2}{ }^{\odot H a n s-C h r i s t o p h ~ L u d w i g, ~}{ }^{4}$ Jutta Gärtner, ${ }^{1}$ \\ and (D) Jens Frahm ${ }^{2,3}$ \\ ${ }^{1}$ Department of Pediatrics and Adolescent Medicine, Division of Pediatric Neurology, University Medical Center Göttingen, 37075 Göttingen, Germany, \\ ${ }^{2}$ Biomedizinische NMR Forschungs GmbH am Max-Planck-Institut für biophysikalische Chemie, 37077 Göttingen, Germany, ${ }^{3} \mathrm{DZHK}$ (German Center for \\ Cardiovascular Research), 37075 Göttingen, Germany, and ${ }^{4}$ Department of Neurosurgery, Division of Pediatric Neurosurgery, University Medical Center \\ Göttingen, 37075 Göttingen, Germany
}

The mechanisms behind CSF flow in humans are still not fully known. CSF circulates from its primary production sites at the choroid plexus through the brain ventricles to reach the outer surface of the brain in the subarachnoid spaces from where it drains into venous bloodstream and cervical lymphatics. According to a recent concept of brain fluid transport, established in rodents, CSF from the brain surface also enters the brain tissue along para-arterial routes and exits through paravenous spaces again into subarachnoid compartments. This unidirectional flow is mainly driven by arterial pulsation. To investigate how CSF flow is regulated in humans, we applied a novel real-time magnetic resonance imaging technique at high spatial $(0.75 \mathrm{~mm})$ and temporal $(50 \mathrm{~ms})$ resolution in healthy human subjects. We observed significant CSF flow exclusively with inspiration. In particular, during forced breathing, high CSF flow was elicited during every inspiration, whereas breath holding suppressed it. Only a minor flow component could be ascribed to cardiac pulsation. The present results unambiguously identify inspiration as the most important driving force for CSF flow in humans. Inspiratory thoracic pressure reduction is expected to directly modulate the hydrostatic pressure conditions for the low-resistance paravenous, venous, and lymphatic clearance routes of CSF. Furthermore, the experimental approach opens new clinical opportunities to study the pathophysiology of various forms of hydrocephalus and to design therapeutic strategies in relation to CSF flow alterations.

Key words: CSF flow; driving force; inspiration; real-time magnetic resonance imaging; respiratory regulation

\section{Introduction}

Although the use of magnetic resonance imaging (MRI) has increased our understanding of human CSF dynamics, the main mechanisms driving CSF flow are still unknown. Brain CSF is contained within subarachnoid spaces and the ventricular system, which is formed by four interconnected cavities (two lateral ventricles, third and fourth ventricles). It circulates from its production site mainly in the choroid plexus of the lateral ventricles through the ventricular system until it reaches the exterior surfaces of brain and spinal cord in the subarachnoid spaces. Over the past decades, several studies used velocity-encoded phase-contrast MRI techniques with cardiac gating to determine quantitative parameters of this flow, such as velocities and stroke volumes. Most reports focus on the pulsatile nature of the CSF flux, which appears to be craniocaudally oriented during cardiac systole and in the reverse direction during diastole (Enzmann and Pelc, 1991; Nitz et al., 1992; Schroth and

\footnotetext{
Received Aug. 5, 2014; revised Nov. 19, 2014; accepted Dec. 8, 2014

Author contributions: S.D.-K., H.-C.L., J.G., and J.F. designed research; S.D.-K., A.A.J., K.-D.M., and J.F. performed research; S.D.-K., A.A.J., K.-D.M., and J.F. analyzed data; S.D.-K., J.G., and J.F. wrote the paper.

This work was supported by the Dorothea Schloezer Program of the Georg August University, Göttingen, Germany (S.D.-K.).

The authors declare no competing financial interests.

Correspondence should be addressed to Steffi Dreha-Kulaczewski, Department of Pediatrics and Adolescent

Medicine, Division of Pediatric Neurology, University Medical Center Göttingen, Robert-Koch-Str. 40, 37075 Göttingen, Germany. E-mail: sdreha@gwdg.de.

DOI:10.1523/JNEUROSCI.3246-14.2015

Copyright $\odot 2015$ the authors $\quad 0270-6474 / 15 / 352485-07 \$ 15.00 / 0$
}

Klose, 1992a; Greitz et al., 1993). Changes in blood volume in the intracranial arteries, its distribution into capillaries, and the resulting oscillations of brain parenchyma have been presumed as main initiating factors of CSF pulsations (HenryFeugeas et al., 2000; Wåhlin et al., 2012). The inward expansion of brain tissue is considered to produce aqueductal flow (Greitz et al., 1994).

In addition, respiration has been shown to influence CSF dynamics via thoracic pressure changes in previous studies using invasive manometer recordings (Williams, 1981). Only very few MRI studies avoided cardiac gating by one-dimensional imaging (Schroth and Klose, 1992b), dynamic echo-planar imaging (Kao et al., 2008), or pencil-beam imaging (Bhadelia et al., 2013) or used a cardiac-gated, spin-labeling method during $6 \mathrm{~s}$ periods of static breathing conditions (Yamada et al., 2013) to confirm a contribution of respiratory rhythms. However, the majority of MRI studies applied phase-contrast flow MRI sequences with synchronization to the electrocardiogram and therefore confined the observation of CSF dynamics to periodic processes in the range of heart rates.

Recent studies in rodents by Illiff et al. $(2012,2013)$ have conceptualized a paravascular pathway system for brain fluids (Strittmatter, 2013; Xie et al., 2013) where the pulsation of penetrating cortical arteries drives CSF from the brain surface along para-arterial routes into the tissue (Iliff et al., 2013) and through paravenous routes out into the venous bloodstream, cervical lymphatics, or CSF spaces (Sakka et al., 2011). 

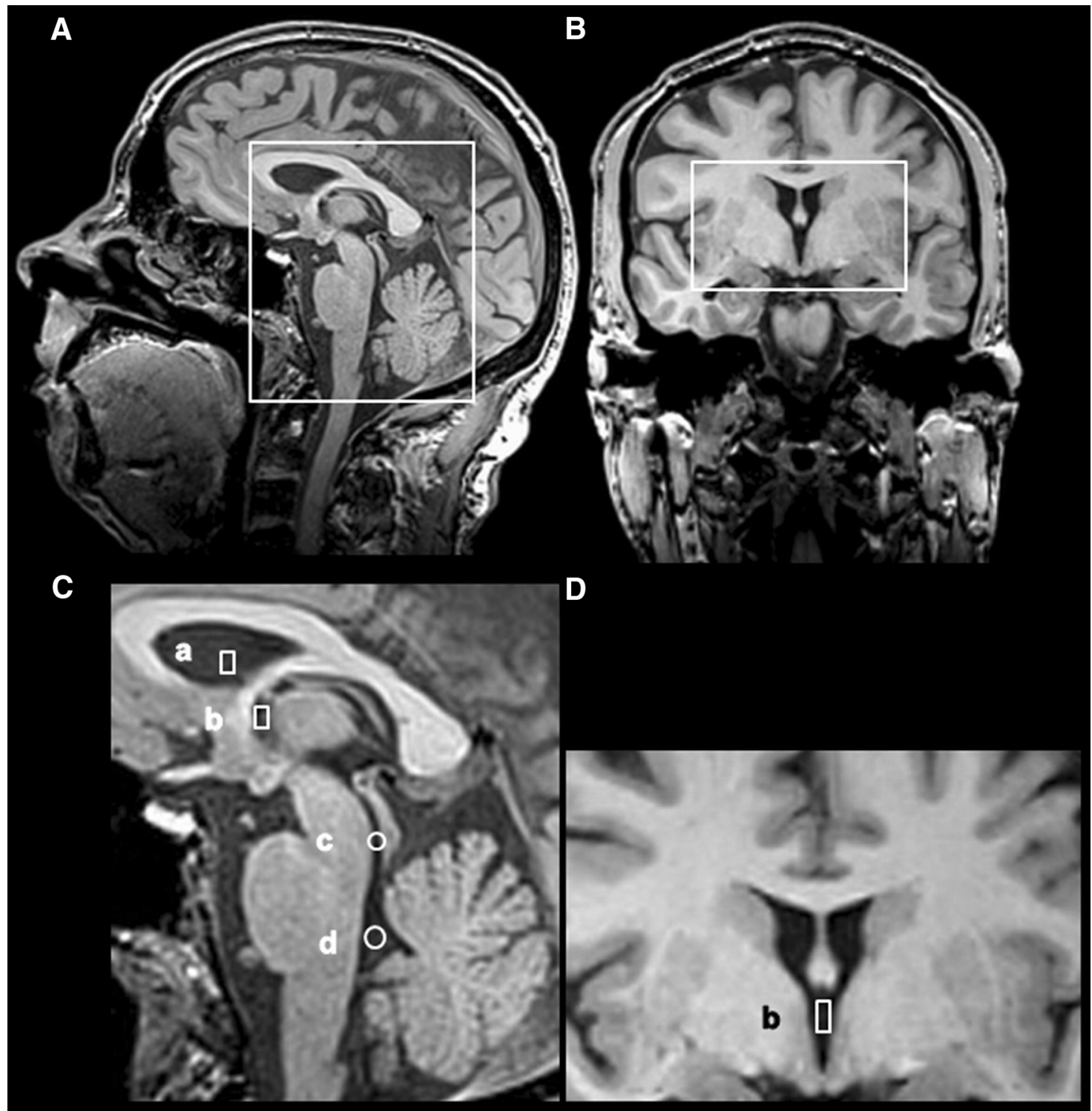

Figure 1. Regions of interest in the CSF ventricular system. Sagittal $(\boldsymbol{A})$ and coronal $(\boldsymbol{B})$ T1-weighted images with boxes indicating respective $(\boldsymbol{C}, \boldsymbol{D})$ magnified representations are shown. Selected regions of interest for the analysis of CSF flow comprise the lateral ventricle (a), third ventricle (b), aqueduct (c), and fourth ventricle (d).

Obviously, the concept of a whole-brain fluid pathway system requires a more comprehensive view on CSF dynamics than provided by a mainly cardiac-driven and rapidly oscillating flow. We applied a novel high-speed MRI technique that concurrently provides both high spatial and temporal resolution to study CSF dynamics in real time, i.e., independent of the assumption of cardiac synchronization and generally independent of repetitive periodic processes. Our results provide unambiguous experimental evidence for inspiration as the key regulator for CSF flow.

\section{Materials and Methods}

We enrolled 11 healthy volunteers, but we had to exclude one subject (female, 25 years) because of poor performance of the breathing protocol. The remaining 10 subjects (age range 19-53 years; two females, eight males) were without contraindication for MRI. The study was approved by the Institutional Review Board, and written informed consent was obtained from each subject before the MRI. This study was in compliance with the Declaration of Helsinki.
MRI was performed at 3T (Tim Trio; Siemens Healthcare) with a standard 32-channel head coil. Real-time MRI was based on a highly undersampled radial gradient-echo sequence with image reconstruction by regularized nonlinear inversion (NLINV) (Uecker et al., 2012; Zhang et al., 2014). The algorithm jointly optimizes the coil sensitivity profiles and the desired image as originally described for Cartesian parallel imaging (Uecker et al., 2008). Subsequent developments achieved true real-time imaging with acquisition times as short as $20 \mathrm{~ms}$ by exploiting the temporal continuity of a dynamic movement (Uecker et al., 2010). Temporal regularization to the preceding frame of an image series adds prior knowledge to the ill-conditioned NLINV reconstruction and effectively constrains the range of possible solutions, i.e., image estimates.

Here, individual T1-weighted images were obtained from sets of 15 spokes per frame acquired within a total measuring time of $50 \mathrm{~ms}$. Specific parameters were as follows: repetition time $=3.33 \mathrm{~ms}$; echo time $=$ $2.10 \mathrm{~ms}$; flip angle $8^{\circ}$; field of view $192 \times 192 \mathrm{~mm}^{2}$; acquisition matrix $256 \times 256$ (no aliasing because of radial acquisitions with twofold oversampling); nominal in-plane resolution $0.75 \times 0.75 \mathrm{~mm}^{2}$; section thickness $5 \mathrm{~mm}$. The spatiotemporal fidelity of the NLINV method was 

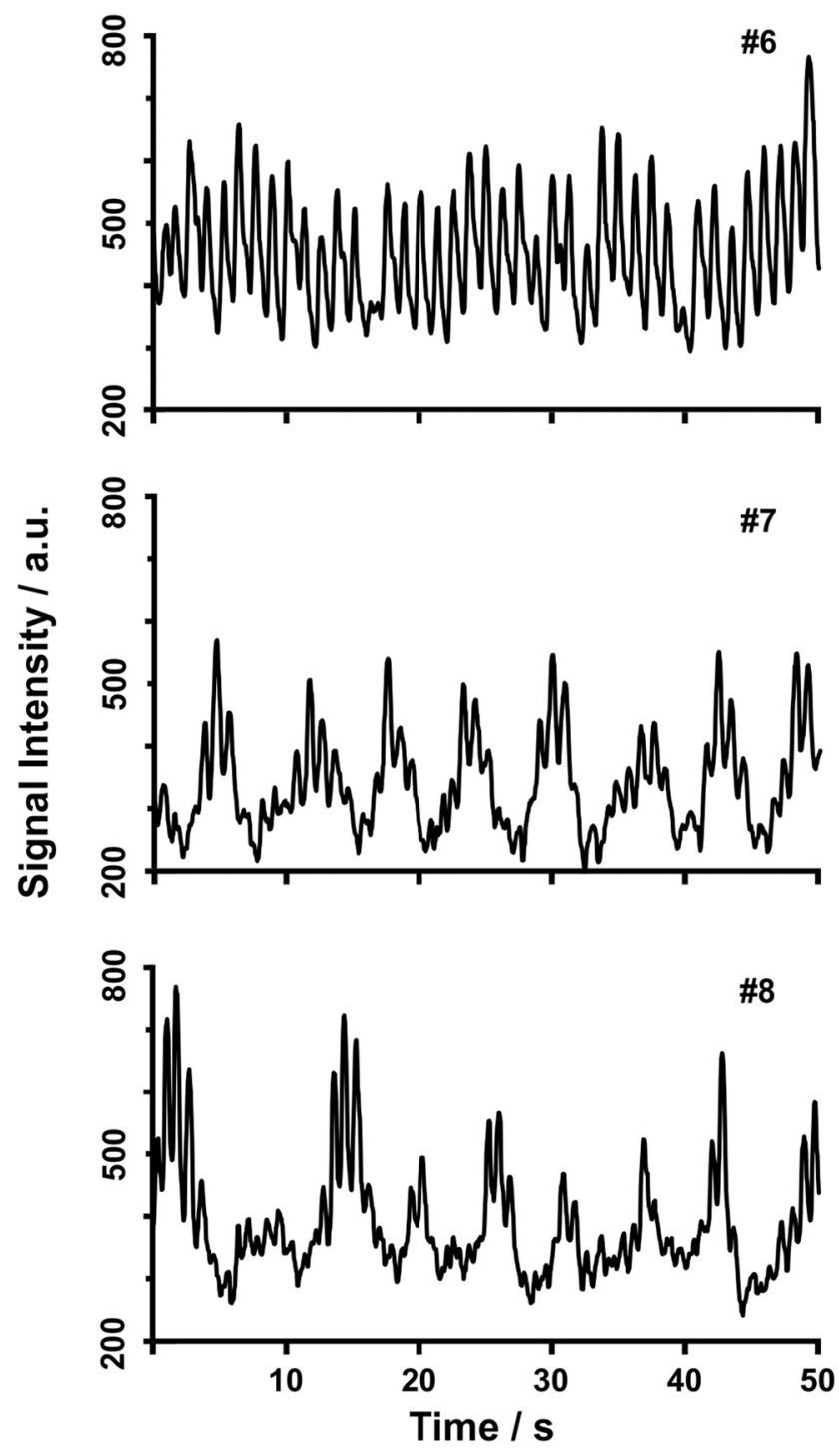

Figure 2. CSF flow in the aqueduct during normal breathing. The traces represent real-time MRI signal intensity time courses at $50 \mathrm{~ms}$ temporal resolution, where relative signal strength (arbitrary units) is proportional to through-plane flow velocity. Although subject 6 (\#6, male, 26 years) presents with pronounced cardiac-related pulsations, subjects 7 ( $\# 7$, male, 43 years) and 8 (\#8, male, 25 years) reveal a stronger, though variable, component that reflects a respiratory modulation of CSF flow.

experimentally validated with the use of a specially designed motion phantom covering a range of image acquisition times and objects moving at different speeds (Frahm et al., 2014).

Measurements during different breathing protocols comprised 1000 images that covered a period of $50 \mathrm{~s}$ duration. Serial images were reconstructed on-line with the use of a highly parallelized version of the NLINV algorithm (Schaetz and Uecker, 2012), which runs on a "bypass" computer (sysGen/TYAN Octuple-GPU, 2x Intel Westmere E5620 processor, 48GB RAM; Sysgen) fully integrated into the host of the MRI system and equipped with two processors (CPUs, SandyBridge E5-2650; Intel) and eight graphical processing units (GPUs, GeForce GTX TITAN; Nvidia).

The flow effect detected by real-time MRI is based on the inflow phenomenon that increases the MRI signal intensity of a T1-weighted image by replacing saturated low signal intensities from stationary tissue or slow-flowing fluids with bright intensities of rapidly flowing fluids. These fresh signal contributions enter the imaging section from neighboring volumes previously unaffected by radiofrequency excitation. The approach results in a monotonous relationship between the relative MRI signal intensity and the flow velocity up to an upper limit where the signal in the selected section ( $5 \mathrm{~mm}$ thickness) is completely replaced by fresh fluid between successive radiofrequency excitations, i.e., within every repetition interval $(3.3 \mathrm{~ms})$ of the MRI acquisition sequence. Accordingly, the sensitivity of this qualitative flow MRI assessment extends to a maximum velocity of $\sim 1.5 \mathrm{~m} \mathrm{~s}^{-1}$.

\section{Results}

Multiple regions of interest (ROIs) along the ventricular system were chosen for CSF flow analyses as indicated in Figure 1. Because most previous studies focused on flow through the aqueduct, i.e., the thin connection between the third and fourth ventricles that closely resembles a vessel, we started our study by evaluating CSF through-plane flow in the same position. As discussed in more detail in Materials and Methods, the T1-weighted real-time MR images exploit the inflow effect of spins flowing perpendicular through the imaging section. This flow replaces low signal intensities from stationary or slow-flowing fluids with brighter intensities from freshly inflowing fluids that originate from neighboring volumes previously unaffected by radiofrequency excitation. Thus, for velocities below a maximum of $\sim 1.5$ $\mathrm{m} \mathrm{s}^{-1}$, the relative MRI signal intensities shown in Figures 2 and 4-6 are proportional to the CSF flow through the imaging section. As demonstrated in Figure 2 for three different subjects, and in agreement with previous reports, we eventually observed a pulsating CSF flow component at a frequency that corresponds to the heart rate. In addition, however, we identified another more significant and indeed dominating component that corresponds to a much stronger CSF flow.

Because measurements in the aqueduct and during normal breathing exhibited a significant variability of the strong component both within and across subjects, we subsequently investigated different positions within the ventricular system (Fig. 1). Moreover, for the main study, we implemented two protocols that required all initial 11 subjects to (1) follow a preset rhythm of forced breathing (eight cycles of $2.5 \mathrm{~s}$ inspiration followed by $2.5 \mathrm{~s}$ expiration each) and (2) hold their breath after inspiration for a period of $12 \mathrm{~s}$. The results in the 10 subjects conducting the first protocol unambiguously reveal that respiratory cycles drive the aforementioned major component of CSF flow. In all cases, measurements in the third ventricle generated the most consistent findings.

Figure 3 illustrates the pronounced MRI signal increase in the third ventricle that is associated with elevated CSF flow during inspiration. Corresponding MRI signal intensity time courses of the entire breathing protocol comprising normal breathing ( $10 \mathrm{~s}$ ) followed by forced inspiration (black boxes $=2.5 \mathrm{~s}$ ) and expiration (for three different subjects and two repetitions each) are depicted in Figure 4 . They clearly demonstrate that exclusively inspiration (Fig. 4, black boxes) elicits significant CSF flow that abates immediately thereafter. In contrast, the cardiac-related CSF flow pattern constituted only a minor contribution in the 10 subjects. This also applies to individual volunteers who presented with a more pronounced cardiac influence (e.g., compare subject 6 in Figs. 2 and 4 during periods of normal breathing). It should also be noted that involuntary head or brain movements were excluded as a source of real-time MRI signal changes. Multiple analyses of a ROI in brain tissue (similar size as in the third ventricle; data not shown) did not reveal any significant motionrelated signal increase.

Additional studies in 10 subjects extended the forced breathing protocol to multiple ROIs along the ventricular system (compare Fig. 1). Selected results for subject 7 are shown 

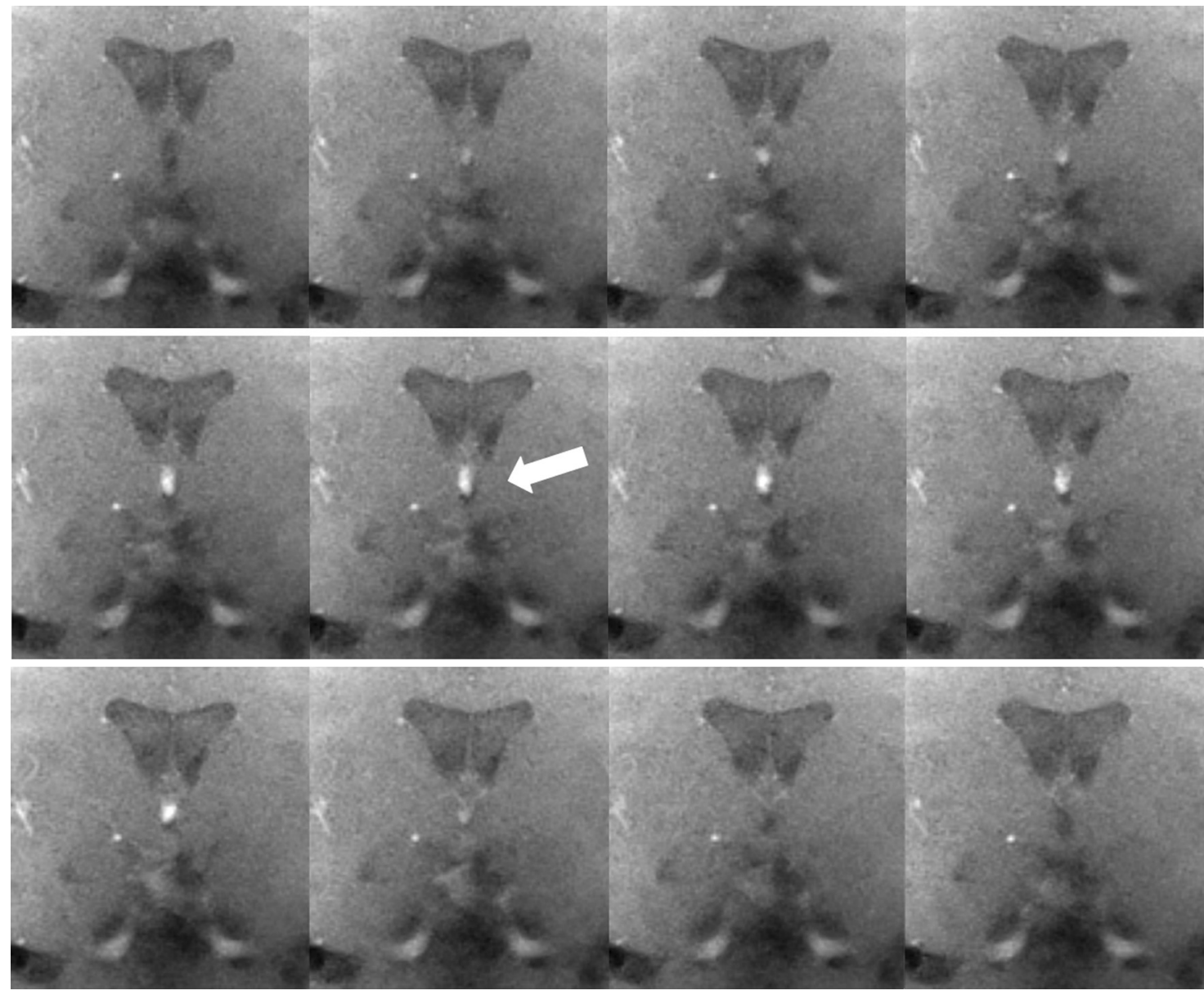

Figure 3. CSF flow in the third ventricle during forced inspiration. The series of images (magnified views) from top left to bottom right was selected from a real-time MRI movie at $50 \mathrm{~ms}$ temporal resolution (every seventh frame, subject 5). The corresponding period of $4.2 \mathrm{~s}$ covers one inspiration $(2.5 \mathrm{~s}$ ) in a coronal view through the third ventricle (compare Fig. 1D). Considerable through-plane flow is demonstrated by the marked MRI signal increase in the third ventricle during inspiration (arrow).

in Figure 5. Despite the observation of slightly higher noise levels for measurements in the lateral ventricle (Fig. $5 a$ ), aqueduct (Fig. 5c), and fourth ventricle (Fig. $5 d$ ) than in the third ventricle (Fig. $5 b$ ), inspiration could generally be identified as the main regulator of CSF flow along the entire axis of the ventricular system. Noteworthy, our data suggest that the aqueduct is the least favorable structure to study CSF flow (Fig. 5). One explanation might be that the aqueduct is exposed to brain movements and is more volatile compared with the third ventricle, which is located in the middle of the brain and framed by sturdy walls.

Finally, complementary control studies involving a $12 \mathrm{~s}$ breath-hold period were performed in 10 subjects. As shown in Figure 6 depicting MRI signal intensity time courses for the corresponding protocol, breath holding entirely suppressed the respiratory-related flow component. Before and after the breathhold period, the protocol requested normal breathing phases that yielded variable CSF flow similar to the initial trials exemplified in Figure 2.

\section{Discussion}

Real-time MRI at adequate spatiotemporal resolution enabled us to study CSF flow in the human brain independent of the assumption of any periodicity. Thus, the present results provide unambiguous evidence that inspiration is the main driving force for CSF dynamics in healthy human subjects, in particular when performed during forced breathing. Consistent with previous studies (Balédent et al., 2004; Flórez et al., 2006; Stadlbauer et al., 2010), we also found a cardiac-related CSF flow component at higher frequency, though at a much lower amplitude and essentially variable across subjects.

Our identification of inspiration as the main mechanism for CSF flow is in contrast to studies that determined cardiac-related pulsations as the most important regulator (Gideon et al., 1994; Strik et al., 2002). However, as far as electrocardiogram-synchronized MRI acquisitions are concerned, this is of no surprise because such methods are unable to detect any nonperiodic processes that are not synchronous to the heart beat. In particular, this applies to slower respiratory modulations of CSF flow. Limitations of our study are mainly attributable to the qualitative assessment of CSF flow via the inflow phenomenon that precludes the determination of absolute velocities and flow directions. However, velocity estimates may be obtained by referencing the CSF-induced signal intensity increases with the inflow-related intensity obtained from blood flow in the superior sagittal sinus, which may be taken from the same imaging section. Because flow in the sagittal sinus yields $\sim 1250$ a.u. for a velocity of $\sim 20 \mathrm{~cm} \mathrm{~s}^{-1}$ compared with $\sim 200-500$ a.u. for CSF flow in the third ventricle during forced inspiration (compare Fig. 4), the resulting CSF velocities are $\sim 3-8 \mathrm{~cm} \mathrm{~s}^{-1}$, depending on the subject and location along the ventricular system. In fact, based on the 


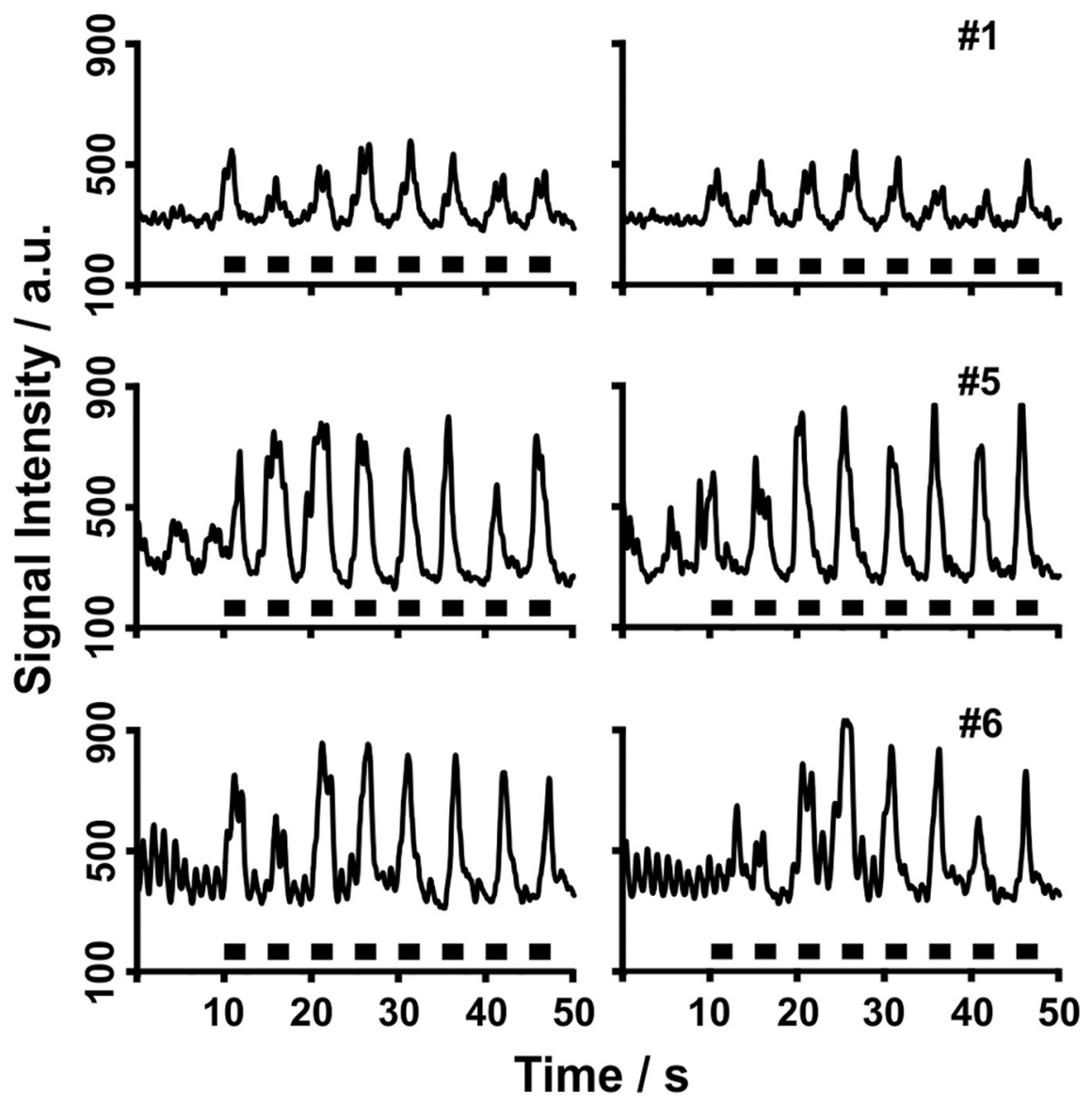

Figure 4. Inspiration drives (SF flow in the third ventricle. MRI signal intensity time courses for subjects 1 (\#1, female, 43 years), 5 ( $\# 5$, male, 19 years), and 6 ( $\# 6$, male, 26 years) for a protocol comprising normal breathing $(10 \mathrm{~s})$ followed by eight periods of forced inspiration (black boxes $=2.5 \mathrm{~s}$ ) and expiration $(2.5 \mathrm{~s}$ ) are shown. The two columns represent repeated measurements for each subject.

present findings, we are now working on a new version of our real-time phase-contrast MRI method previously developed for cardiovascular blood flow (Joseph et al., 2012, 2014). This will allows us to achieve high spatial resolution as well as adequate temporal resolution for directional and quantitative information about inspiration-induced CSF flow velocities and volumes.

Whereas normal breathing elicits strong though variable CSF flow, the remarkable responses to forced respiration point to the reduction of the thoracic pressure during inspiration as the underlying mechanism. Consistent with these results are similar differences of CSF flux in the spinal canal that have been reported between normal and forced breathing conditions using an early one-dimensional MRI technique (Schroth and Klose, 1992b). This also applies to the movements of tagged brain CSF MRI signals recently observed by Yamada et al. (2013) when comparing long periods of inhalation and exhalation.

Originally, the downward and upward CSF flow directions and respective pressure changes along the neuroaxis concomitant to inspiration and expiration have been shown by myelography and invasive manometry (Du Boulay, 1972; Williams, 1981). Respiration-induced changes of thoracic pressure affect the intracranial CSF dynamics by direct exposure of the veins around the thoracic vertebral column, which in turn transmit pressure changes via their abundant anasto- moses into the epidural venous system of the spinal canal. Inspiratory pressure reduction empties the venous plexus and leads to a compensatory CSF flow downward into the spinal canal. Elevated thoracic pressure concurrent with expiration fills the venous plexus and facilitates reverse CSF flow to the head (Sharpey-Schafer, 1965; Williams, 1981). The valveless nature of these venous plexus vessels probably enhances the direct pressure transmission greatly (Henriques, 1962). In addition, the well established link of the CSF compartment to the peripheral lymphatic system (Boulton et al., 1996; Johnston et al., 2004; Koh et al., 2005) implies a connection to respiration as a main constituent of the external lymph pump known to regulate lymphatic backflow to the venous system, in particular from cervical regions (SchmidSchönbein, 1990). This indirect mechanism may provide another contribution to CSF flux that is in line with a respiration-dependent modulation.

Together, the present results indicate that inspiratory thoracic pressure reduction elicits pronounced CSF flow as it is transmitted to the brain subarachnoid spaces via the interconnected venous plexus around the thoracic spinal column and within the spinal canal. Although the present study does not yet resolve whether this dominating component leads a net CSF flow, it seems to act as a modulator of the hydrostatic pressure conditions for the low-resistance CSF clearance routes. This is because inspiration governs venous and lymphatic backflow from the head and neck to the heart. Therefore, apart from cortical arterial pulsation, inspiration may also contribute to the concept of a brain-wide paravascular fluid transport of CSF within the tissue.

In conclusion, real-time MRI of through-plane flow and in response to a predetermined respiration rhythm allows for a noninvasive, rapid, and robust assessment of CSF dynamics in humans. The present results therefore open the door to study the pathophysiology of various forms of hydrocephalus and to design suitable therapeutic approaches. Work is underway to apply this method to studies of flow dynamics in the spinal canal and, furthermore, to patients with various forms of hydrocephalus as a result of disturbed CSF circulation.

\section{Notes}

Supplemental movie 1 for this article is available at https://commons. wikimedia.org/wiki/File\%3ADreha-Kulaczewski_JNeurosci_CSF_ flow_Supplementary_moviel.webm. CSF flow in the third ventricle during forced breathing is shown. The real-time MRI movie is at $50 \mathrm{~ms}$ temporal resolution (subject 5) in a coronal view through the third ventricle during forced breathing: five periods of $2.5 \mathrm{~s}$ of inspiration followed by $2.5 \mathrm{~s}$ of expiration. Considerable CSF flow in the third ventricle is demonstrated by marked MRI signal increases during inspiration. This material has not been peer reviewed. 

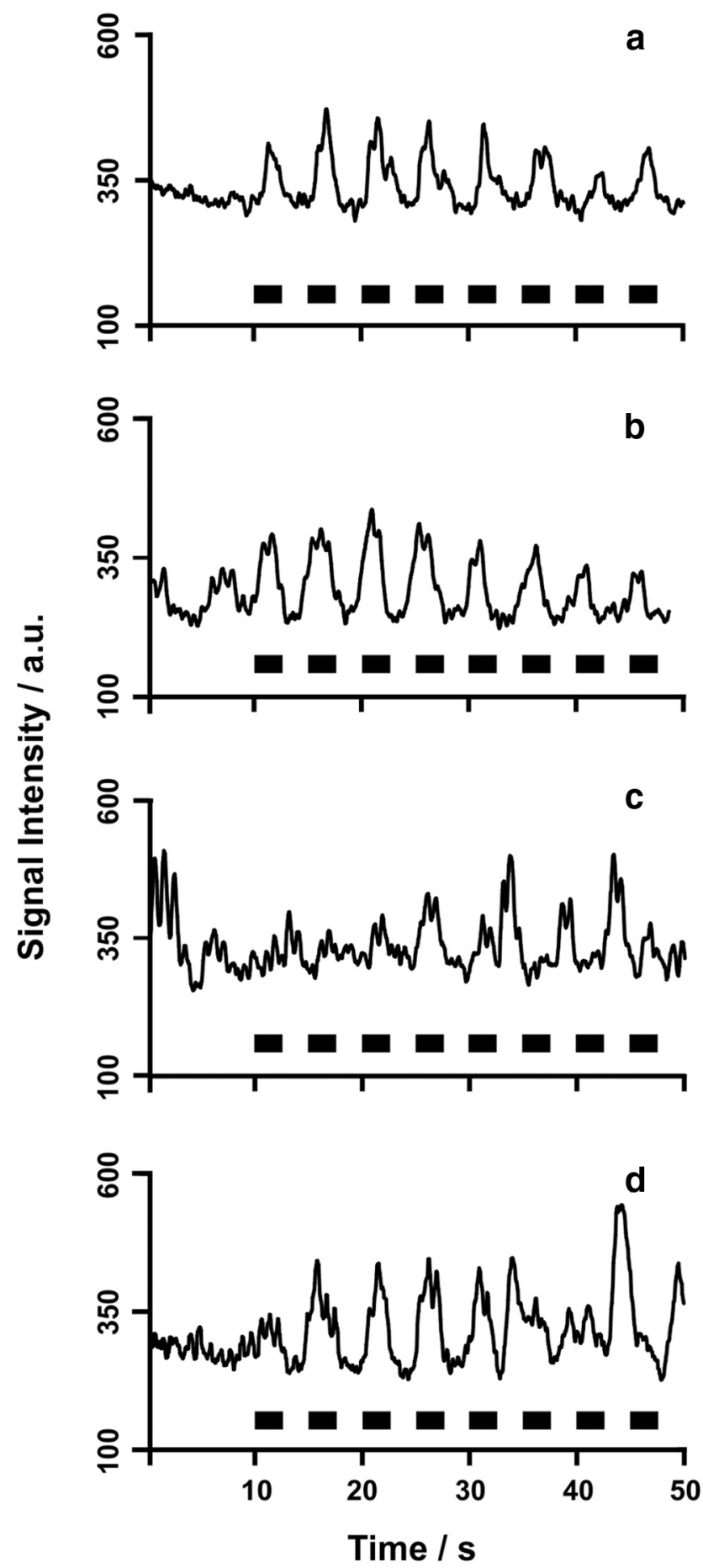

Figure 5. Inspiration drives (SF flow along the entire ventricular system. MRI signal intensity time courses for subject 7 (male, 43 years) for a protocol comprising normal breathing (10 s) followed by forced inspiration (black boxes $=2.5 \mathrm{~s}$ ) and expiration $(2.5 \mathrm{~s})$ are shown. $\boldsymbol{a}$, lateral ventricle; $\boldsymbol{b}$, third ventricle; $\boldsymbol{c}$, aqueduct; $\boldsymbol{d}$, fourth ventricle (compare Fig. 1 ).

\section{References}

Balédent O, Gondry-Jouet C, Meyer ME, De Marco G, Le Gars D, HenryFeugeas MC, Idy-Peretti I (2004) Relationship between cerebrospinal fluid and blood dynamics in healthy volunteers and patients with communicating hydrocephalus. Invest Radiol 39:45-55. CrossRef Medline

Bhadelia RA, Madan N, Zhao Y, Wagshul ME, Heilman C, Butler JP, Patz S (2013) Physiology-based MR imaging assessment of CSF flow at the foramen magnum with a Valsalva maneuver. AJNR Am J Neuroradiol 34: 1857-1862. CrossRef Medline
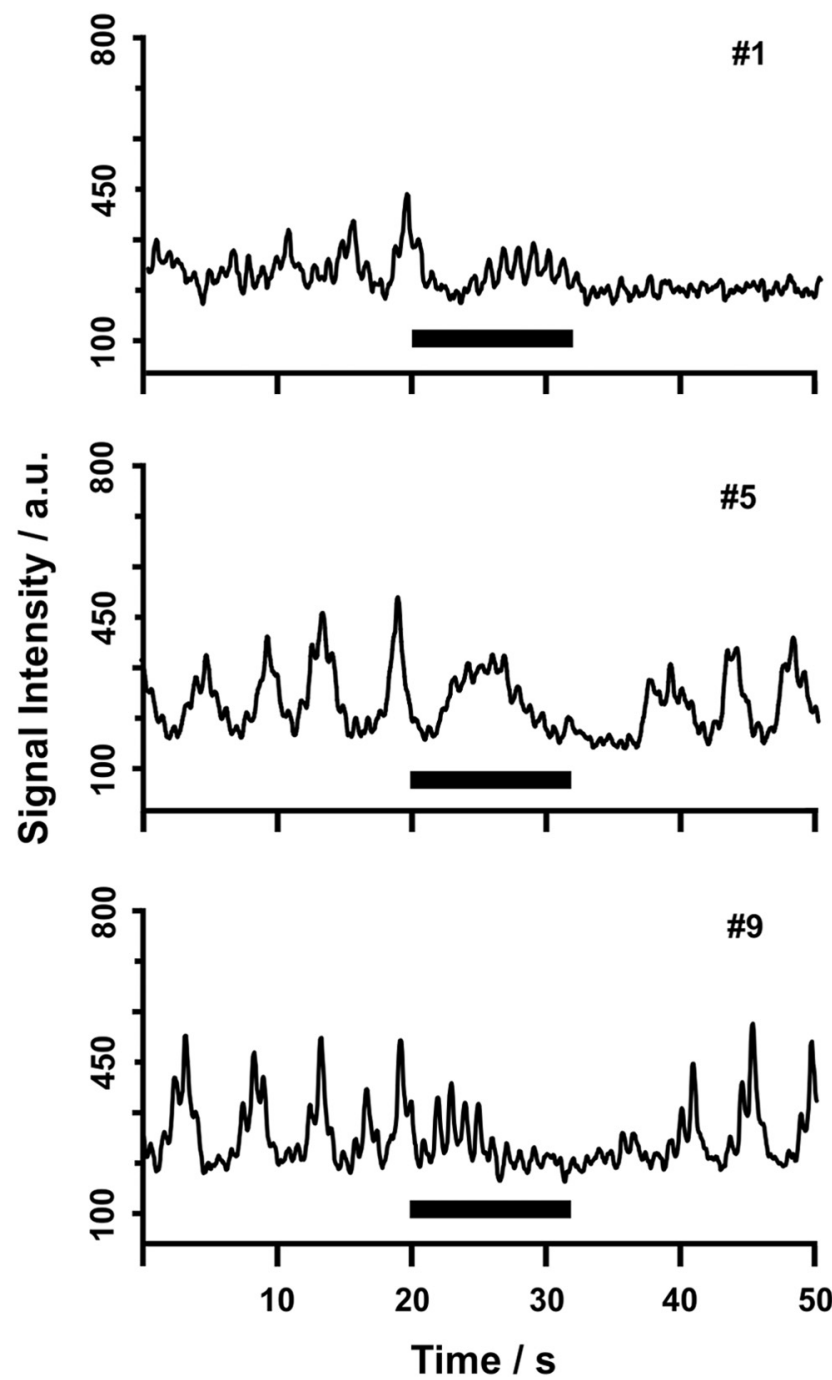

Figure 6. Breath holding abolishes (SF flow in the third ventricle. MRI signal intensity time courses for subjects 1 ( $\# 1$, female, 43 years), 5 ( $\# 5$, male, 19 years), and 9 ( $\#$, male, 22 years) for a protocol comprising normal breathing and intermediate breath holding after inspiration (black bar $=12 \mathrm{~s}$ ) are shown.

Boulton M, Young A, Hay J, Armstrong D, Flessner M, Schwartz M, Johnston M (1996) Drainage of CSF through lymphatic pathways and arachnoid villi in sheep: measurement of 125I-albumin clearance. Neuropathol Appl Neurobiol 22:325-333. CrossRef Medline

Du Boulay G (1972) Specialisation broadens the view. The significance of a CSF pulse. Clin Radiol 23:401-409. CrossRef Medline

Enzmann DR, Pelc NJ (1991) Normal flow patterns of intracranial and spinal cerebrospinal fluid defined with phase-contrast cine MR imaging. Radiology 178:467-474. CrossRef Medline

Flórez YN, Moratal D, Forner J, Martí-Bonmatí L, Arana E, GuajardoHernández U, Millet-Roig J (2006) Semiautomatic analysis of phase contrast magnetic resonance imaging of cerebrospinal fluid flow through the aqueduct of Sylvius. MAGMA 19:78-87. CrossRef Medline

Frahm J, Schaetz S, Untenberger M, Zhang S, Voit D, Merboldt KD, Sohns JM, Lotz J, Uecker M (2014) On the temporal fidelity of nonlinear inverse reconstructions for real-time MRI-the motion challenge. Open Med Imaging J 8:1-7. CrossRef

Gideon P, Sørensen PS, Thomsen C, Ståhlberg F, Gjerris F, Henriksen O (1994) Assessment of CSF dynamics and venous flow in the superior sagittal sinus by MRI in idiopathic intracranial hypertension: a preliminary study. Neuroradiology 36:350-354. CrossRef Medline

Greitz D, Franck A, Nordell B (1993) On the pulsatile nature of intracranial and spinal CSF-circulation demonstrated by MR imaging. Acta Radiol 34:321-328. Medline 
Greitz D, Hannerz J, Rähn T, Bolander H, Ericsson A (1994) MR imaging of cerebrospinal fluid dynamics in health and disease. On the vascular pathogenesis of communicating hydrocephalus and benign intracranial hypertension. Acta Radiol 35:204-211. Medline

Henriques CQ (1962) The veins of the vertebral column and their role in the spread of cancer. Ann R Coll Surg Engl 31:1-22. Medline

Henry-Feugeas MC, Idy-Peretti I, Baledent O, Poncelet-Didon A, Zannoli G, Bittoun J, Schouman-Claeys E (2000) Origin of subarachnoid cerebrospinal fluid pulsations: a phase-contrast MR analysis. Magn Reson Imaging 18:387-395. CrossRef Medline

Iliff JJ, Wang M, Liao Y, Plogg BA, Peng W, Gundersen GA, Benveniste H, Vates GE, Deane R, Goldman SA, Nagelhus EA, Nedergaard M (2012) A paravascular pathway facilitates CSF flow through the brain parenchyma and the clearance of interstitial solutes, including amyloid $\beta$. Sci Transl Med 4:147ra111. CrossRef Medline

Iliff JJ, Wang M, Zeppenfeld DM, Venkataraman A, Plog BA, Liao Y, Deane R, Nedergaard M (2013) Cerebral arterial pulsation drives paravascular CSF-interstitial fluid exchange in the murine brain. J Neurosci 33:1819018199. CrossRef Medline

Johnston M, Zakharov A, Papaiconomou C, Salmasi G, Armstrong D (2004) Evidence of connections between cerebrospinal fluid and nasal lymphatic vessels in humans, non-human primates and other mammalian species. Cerebrospinal Fluid Res 1:2-15. CrossRef Medline

Joseph AA, Merboldt KD, Voit D, Zhang S, Uecker M, Lotz J, Frahm J (2012) Real-time phase-contrast MRI of cardiovascular blood flow using undersampled radial FLASH and nonlinear inverse reconstruction. NMR Biomed 25:917-924. CrossRef Medline

Joseph A, Kowallick JT, Merboldt KD, Voit D, Schaetz S, Zhang S, Sohns JM, Lotz J, Frahm J (2014) Real-time flow MRI of the aorta at a resolution of 40 ms. J Magn Reson Imaging 40:206-213. CrossRef Medline

Kao YH, Guo WY, Liou AJ, Hsiao YH, Chou CC (2008) The respiratory modulation of intracranial cerebrospinal fluid pulsation observed on dynamic echo planar images. Magn Reson Imaging 26:198-205. CrossRef Medline

Koh L, Zakharov A, Johnston M (2005) Integration of the subarachnoid space and lymphatics: is it time to embrace a new concept of cerebrospinal fluid absorption? Cerebrospinal Fluid Res 2:6-17. CrossRef Medline

Nitz WR, Bradley WG Jr, Watanabe AS, Lee RR, Burgoyne B, O'Sullivan RM, Herbst MD (1992) Flow dynamics of cerebrospinal fluid: assessment with phase-contrast velocity MR imaging performed with retrospective cardiac gating. Radiology 183:395-405. CrossRef Medline

Sakka L, Coll G, Chazal J (2011) Anatomy and physiology of cerebrospinal fluid. Eur Ann Otorhinolaryngol Head Neck Dis 128:309-316. CrossRef Medline

Schaetz S, Uecker M (2012) A multi-GPU programming library for realtime applications. Lect Notes Comp Sci 7439:114-128. CrossRef
Schmid-Schönbein GW (1990) Microlymphatics and lymph flow. Physiol Rev 70:987-1028. Medline

Schroth G, Klose U (1992a) Cerebrospinal fluid flow. I. Physiology of cardiac-related pulsation. Neuroradiology 35:1-9. CrossRef Medline

Schroth G, Klose U (1992b) Cerebrospinal fluid flow. II. Physiology of respiration-related pulsations. Neuroradiology 35:10-15. CrossRef Medline

Sharpey-Schafer EP (1965) Effects of respiratory acts on the circulation. In: Physiology (Hamilton WF, Dow P eds), pp 1875-1886. Baltimore: Waverly.

Stadlbauer A, Salomonowitz E, van der Riet W, Buchfelder M, Ganslandt O (2010) Insight into the patterns of cerebrospinal fluid flow in the human ventricular system using MR velocity mapping. Neuroimage 51:42-52. CrossRef Medline

Strik C, Klose U, Erb M, Strik H, Grodd W (2002) Intracranial oscillations of cerebrospinal fluid and blood flows: analysis with magnetic resonance imaging. J Magn Reson Imaging 15:251-258. CrossRef Medline

Strittmatter WJ (2013) Bathing the brain. J Clin Invest 123:1013-1015. CrossRef Medline

Uecker M, Hohage T, Block KT, Frahm J (2008) Image reconstruction by regularized nonlinear inversion-joint estimation of coil sensitivities and image content. Magn Reson Med 60:674-682. CrossRef Medline

Uecker M, Zhang S, Voit D, Karaus A, Merboldt KD, Frahm J (2010) Realtime MRI at a resolution of 20 ms. NMR Biomed 23:986-994. CrossRef Medline

Uecker M, Zhang S, Voit D, Merboldt KD, Frahm J (2012) Real-time MRIrecent advances using radial FLASH. Imaging Med 4:461-476. CrossRef

Wåhlin A, Ambarki K, Hauksson J, Birgander R, Malm J, Eklund A (2012) Phase contrast MRI quantification of pulsatile volumes of brain arteries, veins, and cerebrospinal fluids compartments: repeatability and physiological interactions. J Magn Reson Imaging 35:1055-1062. CrossRef Medline

Williams B (1981) Simultaneous cerebral and spinal fluid pressure recordings. I. Technique, physiology, and normal results. Acta Neurochir (Wien) 58:167-185. CrossRef

Xie L, Kang H, Xu Q, Chen MJ, Liao Y, Thiyagarajan M, O’Donnell J, Christensen DJ, Nicholson C, Iliff JJ, Takano T, Deane R, Nedergaard M (2013) Sleep drives metabolite clearance from the adult brain. Science 342:373-377. CrossRef Medline

Yamada S, Miyazaki M, Yamashita Y, Ouyang C, Yui M, Nakahashi M, Shimizu S, Aoki I, Morohoshi Y, McComb JG (2013) Influence of respiration on cerebrospinal fluid movement using magnetic resonance spin labeling. Fluids Barriers CNS 10:36-44. CrossRef Medline

Zhang S, Joseph AA, Voit D, Schaetz S, Merboldt KD, Unterberg-Buchwald C, Hennemuth A, Lotz J, Frahm J (2014) Real-time magnetic resonance imaging of cardiac function and flow-recent progress. Quant Imaging Med Surg 4:313-329. CrossRef Medline 